\title{
Chasing extreme blazars with INTEGRAL
}

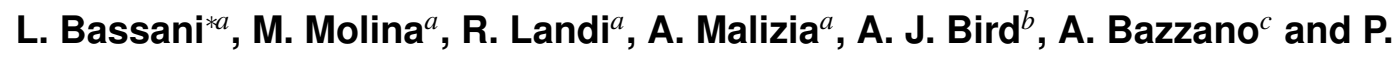 \\ Ubertini $^{c}$ \\ a INAF/IASF Bologna, Italy \\ ${ }^{b}$ School of Physics and Astronomy, University of Southampton, UK \\ ${ }^{c}$ INAF/IAPS Rome, Italy \\ E-mail: bassani@iasfbo.inaf.it
}

\begin{abstract}
Within the blazar population, hard X-ray selected objects are of particular interest as they tend to lie at each end of the blazar sequence. In particular, flat spectrum radio quasars located at high redshifts display the most powerful jets, the largest black hole masses and the most luminous accretion disks: their spectral energy distribution has a Compton peak in the sub-MeV region which favours their detection by instruments like INTEGRAL/IBIS and Swift/BAT. These sources are even more extreme than blazars selected in other wavebands, like, for example, the gammaray range explored by Fermi. Here we report on a sample of 12 high redshift blazars detected so far by INTEGRAL, including 3 newly identified objects. Some properties of the combined IBIS/BAT sample of high redshift blazars $(\mathrm{z} \geq 2)$ are also compared to those of a similar similar sample obtained by Fermi.
\end{abstract}

An INTEGRAL view of the high-energy sky (the first 10 years) - 9th INTEGRAL Workshop and celebration of the 10th anniversary of the launch

15-19 October 2012

Bibliotheque Nationale de France, Paris, France

${ }^{*}$ Speaker. 


\section{Introduction}

Among Active Galactic Nuclei, the most extreme and powerful objects are blazars. In the commonly accepted scenario of blazars, a single population of high-energy electrons in a relativistic jet radiate from the radio/FIR to the UV/soft X-rays through synchrotron radiation and, at higher frequencies, through inverse Compton (IC), scattering soft-target photons present either in the jet (synchrotron self-Compton [SSC] model), in the surrounding material (external Compton [EC] model), or in both [8]. In fact, the Spectral Energy Distribution (SED) of blazars displays two peaks: the synchrotron one (from the IR to the soft X-rays) and the Compton peak ( $\mathrm{MeV} / \mathrm{GeV}$ band). The different SEDs observed in blazars can be explained through the blazar sequence [7], which proposes that the more luminous sources have both synchrotron and Compton peaks at lower energies than their fainter (and generally at lower redshifts) counterparts. Recently, [9] showed that hard X-ray selected blazars at high $\mathrm{z}$ are those with the most powerful jets, the most luminous accretion disks and the largest black hole masses, i.e. they are even more extreme than blazars selected in gamma-rays. This can be understood in terms of the blazar sequence, since the high energy peak in the SED of the most powerful blazars is in the hard $\mathrm{X}$-ray/MeV range. As a consequence, these objects are more luminous in the $20-100 \mathrm{keV}$ band than above $100 \mathrm{MeV}$, and thus become detectable in hard X-ray surveys even if they are undetected in gamma-ray ones. Thus, a hard $\mathrm{X}$-ray survey is more efficient in finding the most powerful blazars lying at the highest $\mathrm{z}$. Here we present a sample of 12 high redshift blazars $(\mathrm{z}>1$ ) detected by INTEGRAL, including three newly reported sources, and discuss their overall properties.

\section{The Blazar Sample}

The sample consists of 12 high redshift blazars: 9 from [11] and 1 from [10], while 2 have been fully discussed by [3] and [14] respectively; it covers a redshift range from 1 to almost 4 . The table reports the main properties of these objects: redshift, hard and soft X-ray flux, black hole mass, $5 \mathrm{GHz}$ flux as well as detection by either Fermi or BAT or both. The sources in the sample are characterised by 20-100 keV luminosities spanning from about $10^{46}$ to $10^{48} \mathrm{erg} \mathrm{s}^{-1}$ and black hole masses from $12 \times 10^{7}$ to at least $5 \times 10^{9}$ solar masses; note also that all have a $5 \mathrm{GHz}$ radio flux $>0.1$ Jy. Three (IGR J06073-0024, IGR J12319-0749, IGR J17438-0347) of this 12 objects have only recently been identified and in the following we will summarise their main properties.

IGR J06073-0024 is optically classified as a broad emission line AGN (FWHM $=5600 \mathrm{~km} \mathrm{~s}^{-1}$ ) at $\mathrm{z}=1.028$ [12]; this source has a counterpart in the CRATES catalogue with a $8.4 \mathrm{GHz}$ flux of $\sim 98 \mathrm{mJy}$ and is also reported in the NVSS survey with a $1.4 \mathrm{GHz}$ flux of $133.9 \mathrm{mJy}$, yielding to a flat radio spectral index of -0.15 . The statistical quality of the XRT spectrum is such that only an estimate of the $2-10 \mathrm{keV}$ flux is available $\left(\mathrm{F}_{2-10 \mathrm{keV}}=1.1 \times 10^{-13} \mathrm{erg} \mathrm{cm}^{2} \mathrm{~s}^{-1}\right)$. At the reported redshift and assuming $\mathrm{H}_{0}=71 \mathrm{~km} \mathrm{~s}^{-1} \mathrm{Mpc}^{-1}, \Omega_{\Lambda}=0.73$ and $\Omega_{\mathrm{M}}=0.27$, the source X-ray luminosities are $3.8 \times 10^{43} \mathrm{erg} \mathrm{s}^{-1}$ and $4.1 \times 10^{45} \mathrm{erg} \mathrm{s}^{-1}$ in the $2-10 \mathrm{keV}$ and $20-40 \mathrm{keV}$ band, respectively; this suggests that in IGR J06073-0024 the X-ray spectrum rises towards higher frequencies, as expected in flat spectrum radio quasars (see also [14] for details). The mass of the black hole at the centre of this object has been estimated to be of the order of $5 \times 10^{8} \mathrm{M}_{\odot}$ by [12]. 


\begin{tabular}{|c|c|c|c|c|c|c|c|}
\hline Name & $\mathbf{z}$ & $\mathbf{F}_{20-100 \mathrm{keV}}$ & $\overline{\mathbf{F}_{2-10 \mathrm{keV}}^{\ddagger}}$ & $\begin{array}{c}\text { Mass } \\
\left(\mathbf{1 0}^{9} \mathbf{M}_{\odot}\right) \\
\end{array}$ & $\begin{array}{l}\mathbf{F}_{5 \mathrm{GHz}} \\
(\mathbf{m J y})\end{array}$ & Fermi/BAT $^{\dagger}$ & Alt. Name \\
\hline Swift J0218.0+7348 & 2.367 & 2.70 & 0.55 & 0.01 & 2278 & Fermi/BAT & S5 0212+73 \\
\hline NRAO 140 & 1.258 & 2.46 & 0.72 & 1.8 & 1960 & Fermi/BAT & 4C 32.14 \\
\hline PKS $0528+134$ & 2.06 & 1.50 & 2.57 & 1 & 1960 & Fermi/BAT & - \\
\hline QSO 0539-2839 & 3.104 & 1.74 & 0.17 & 2 & 1278 & Fermi/BAT & PKS 0537-286 \\
\hline QSO $0836+710$ & 2.218 & 5.77 & 2.63 & 3 & 2440 & Fermi/BAT & 4C 71.07 \\
\hline Swfit J1656.3-3302 & 2.40 & 3.04 & 0.44 & 2.6 & 290 & Fermi/BAT & - \\
\hline PKS 1830-211 & 2.507 & 4.83 & 1.00 & 1.60 & 5870 & Fermi/BAT & - \\
\hline PKS 2149-306 & 2.345 & 2.51 & 0.80 & 5 & 1720 & Fermi/BAT & - \\
\hline IGR J22517+2218 & 3.668 & 3.15 & 0.26 & 1 & 174 & -/BAT & - \\
\hline \multicolumn{8}{|c|}{ Extra Sources } \\
\hline IGR J06073-0024 & 1.08 & $<3.28$ & 0.01 & 0.5 & $96 / 116$ & - & PMN J0606-0025 \\
\hline IGR J12319-0749 & 3.1 & 0.83 & 0.33 & 2.8 & - & - & - \\
\hline IGR J17438-0347 & 1.05 & 0.8 & 1.92 & 3.7 & 2300 & - & PKS 1741-03 \\
\hline
\end{tabular}

IGR J12319-0734 is optically classified as a broad emission line AGN (FWHM $=5600 \mathrm{~km}$ $\mathrm{s}^{-1}$ ) at $\mathrm{z}=3.1$ by [12]; the mass of the central black hole is estimated to be $2.8 \times 10^{9} \mathrm{M}_{\odot}$. The joint spectral analysis of the XRT and IBIS data [3] indicates that the soft X-ray spectrum is hard and bright: a simple power law provides an acceptable fit with $\Gamma=1.35 \pm 0.14$. Since in the data to model ratio there is some evidence for a high energy cut-off $\left(\mathrm{E}_{\mathrm{cut}}\right)$, the broad-band spectrum has also been fitted with a cut-off power law, yielding $\Gamma=1.24_{-0.19}^{+0.17}$ and $\mathrm{E}_{\text {cut }}=24.5_{-15}^{+71} \mathrm{keV}$. Note that the cross-calibration constant between the two instruments, introduced in the fit to take into account possible flux and/or instrumental mismatches between the two datasets, is $\mathrm{C}=2.53_{-1.55}^{+5.24}$, indicating some degree of flux variability. Assuming that this broad-band fit represents the average state of the source, the estimated observer frame luminosities are $2.8 \times 10^{47} \mathrm{erg} \mathrm{s}^{-1}$ in the X-ray $(2-10 \mathrm{keV})$ band and $7.3 \times 10^{47} \mathrm{erg} \mathrm{s}^{-1}$ in the $20-100 \mathrm{keV}$ band, i.e. IGR J12319-0749 has a high energy luminosity similar to those observed in the [9] sample of high redshifts blazars. The source SED (see [3] for details) resembles that of a blazar with the synchrotron peak located between radio and near-infrared frequencies and the Compton peak in the hard X-ray band, as also suggested by the break measured in the combined XRT/IBIS spectrum.

IGR J17438-0347, or PKS 1741-03, [10] is a quasar at $\mathrm{z}=1.054$. First considered as one of the EGRET sources detected with high confidence [13], IGR J17438-0347 was not reported in a subsequent re-analysis of EGRET data [5] nor has been seen by Fermi so far. It is a strong (2.3 Jy at $5 \mathrm{GHz}$ ) radio source with a flat core spectrum, dominating the radio emission. The source displays variability when monitored over the $5-22 \mathrm{GHz}$ range [1]. The black hole mass found for this object is again above $10^{9} \mathrm{M}_{\odot}$ [6]. The X-ray spectrum obtained by the XRT instrument is well fitted by a simple power-law with a flat photon index $(\Gamma \sim 1.4)$ and absorbed by a mild intrinsic column density $\left(\mathrm{N}_{\mathrm{H}} \sim 0.6 \times 10^{22} \mathrm{~cm}^{-2}\right)$. It is possible that the lack of counts at low energies is due to absorption local to the source or to intrinsic spectral curvature, as observed in other high z blazars [2]. The $2-10 \mathrm{keV}$ luminosity is $5.61 \times 10^{46} \mathrm{erg} \mathrm{s}^{-1}$, while the $20-10 \mathrm{keV}$ luminosity is $2.34 \times 10^{46} \mathrm{erg} \mathrm{s}^{-1}$.

\section{Conclusions}

So far 17 blazars at redshift higher than 2 have been detected above $10 \mathrm{keV}$ by either INTEGRAL/IBIS (this work) or Swift/BAT [4] or both; of these, 10 are above z $=2$ and, even more 

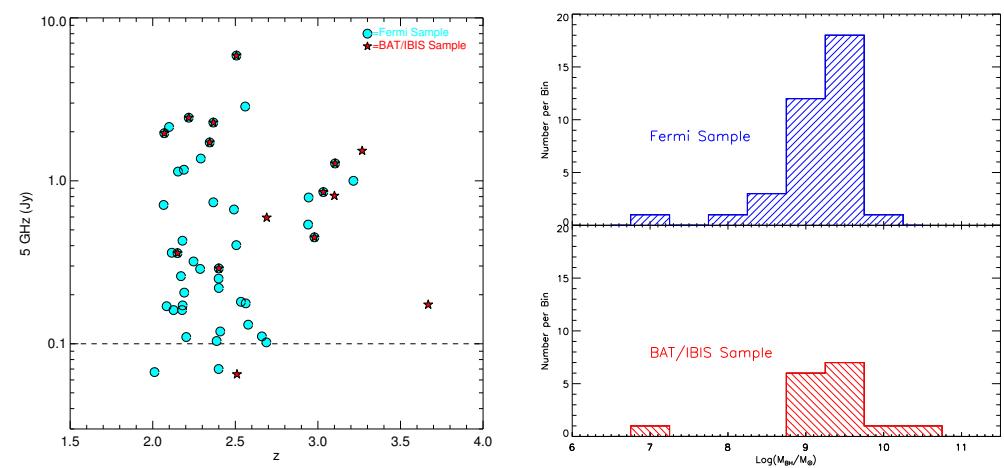

Figure 1: Left Panel: $\mathrm{F}_{5 \mathrm{GHz}} v s . \mathrm{z}$ for the blazar samples detected by Fermi (cyan circles) and by IBIS/BAT (red stars). Right Panel: distribution of black hole masses for blazars in the Fermi sample (blue histogram) and in the IBIS/BAT sample (red histogram).

interesting, 7 are above $\mathrm{z}=3$. Figures 1 compare the sample of distant $(\mathrm{z}>2)$ blazars detected by Fermi with that seen by IBIS and BAT: it is evident that observations in hard X-rays allowed the detection of objects at high redshifts (Figure 1 left panel) and with large black hole masses (Figure 1 right panel), with the same or even better capability than Fermi. Note also that all the high $\mathrm{z}$ blazars so far detected at high energies have a $5 \mathrm{GHz}$ flux $\geq 0.1 \mathrm{Jy}$, making this threshold a possible way of selecting high $\mathrm{z}$ blazars in hard X-ray surveys. As the exploration of the hard $\mathrm{X}$-ray sky continues, it is expected that more high redshift blazars with similar characteristics to those presented here will be found and studied.

\section{References}

[1] Bach U., Raiteri C. M., Villata M. 2007, A\&A, 464, 175

[2] Bassani L., Landi R., Malizia A. et al. 2007, ApJL, 669, 1

[3] Bassani L., Landi R., Marshall F. E. et al. 2012, A\&A, 543, 1

[4] Baumgartner W. H., Tueller J., Markwardt C., Skinner G. 2010, HEAD, 11, 1305

[5] Casandjian J. M. \& Grenier I. A. 2008, A\&A, 489, 849

[6] Fan Z.-H. \& Cao, X. 2004, ApJ, 602, 103

[7] Fossati G., Maraschi L., Celotti A. et al. 1998, MNRAS, 29, 433

[8] Ghisellini G., Celotti A., Fossati G. et al. 1998, MNRAS, 301, 451

[9] Ghisellini G., Della Ceca R., Volonteri M. et al. 2010, MNRAS, 405, 387

[10] Krivonos R., Tsygankov S., Lutovinov A. et al. 2012, A\&A, 545, 27

[11] Malizia A., Bassani L., Bazzano A. et al. 2012, MNRAS, 426, 1750

[12] Masetti N., Parisi P., Jiménez-Bailón E. et al. 2012, A\&A, 538, 123

[13] Mattox J. R., Hartman R. C., Reimer O. 2001, ApJS, 135, 155

[14] Molina M., Landi R., Bassani L. et al. 2012, A\&A, 548, 32 